CERN-TH/2000-256

SNUST-000801

TIFR/TH/00-48

hep-th/0008214

\title{
A Note on D-Branes of Odd Codimensions From Noncommutative Tachyons
}

\author{
Gautam Mandal ${ }^{a, b}$ and Soo-Jong Rey ${ }^{a, c}$ \\ Theory Division, CERN, CH-1211, Genevé, Switzerland ${ }^{a}$ \\ Tata Institute for Fundamental Research \\ Homi Bhabha Road, Mumbai 400005 India $^{b}$ \\ School of Physics 83 Center for Theoretical Physics \\ Seoul National University, Seoul 151-747 Korea ${ }^{c}$ \\ mandal@theory.tifr.res.in \\ sjrey@gravity.snu.ac.kr
}

\begin{abstract}
On a noncommutative space of rank-1, we construct a codimension-one soliton explicitly and, in the context of noncommutative bosonic open string field theory, identify it with the D24brane. We compute the tension of the proposed D24-brane, yielding an exact value and show that it is related to the tension of the codimension-two D23-brane by the string T-duality. This resolves an apparent puzzle posed by the result of Harvey, Kraus, Larsen and Martinec and proves that the T-duality is a gauge symmetry; in particular, at strong noncommutativity, it is part of the $\mathrm{U}(\infty)$ gauge symmetry on the worldvolume. We also apply the result to non-BPS D-branes in superstring theories and argue that the codimension-one soliton gives rise to new descent relations among the non-BPS D-branes in Type IIA and Type IIB string theories via T-duality.
\end{abstract}

\footnotetext{
${ }^{1}$ Work supported in part by BK-21 Initiative in Physics (SNU - Project 2), KRF International Collaboration Grant, KOSEF Interdisciplinary Research Grant 98-07-02-07-01-5, and KOSEF Leading Scientist Grant.
} 
In a series of recent important developments [1, 2, 3], it has been noted that noncommutative field theories in various dimensions arise quite naturally at various corners of the moduli space of nonperturbative string theories, especially, when a nonzero value of the NS two-form potential $B_{2}$ is turned on, inducing noncommutativity on the open string dynamics.

Among the most interesting developments are noncommutative solitons, in particular, in the context of the string theories. At infinite noncommutativity limit, Gopakumar, Minwalla and Strominger [4] have constructed explicit forms of static solitons by utilizing an isomorphism between fields on noncommutative plane and operators on a single-particle Hilbert space, $\mathcal{H}_{\theta}$ — viz. the Weyl-Moyal correspondence]. The method of [4] were adapted by Harvey, Kraus, Larsen and Martinec [6] and Dasgupta, Mukhi and Rajesh [7] to bosonic string and superstring theories, respectively, and have constructed a noncommutative version of D-branes, supporting the viewpoint that D-branes are built out of solitonic lumps of tachyon living on higher dimensional D-branes [10]. The result of [6, [] has an important implication for Sen's conjecture [9] concerning universality of the tachyon potential in that, in the limit of infinitely strong noncommutativity the level-zero truncation yields exactly Sen's conjectural relation between the D-brane tension $T_{\mathrm{D}}$ and the height of the tachyon potential $V(T)$ :

$$
T_{\mathrm{D}}+\left(V(0)-V\left(T_{0}\right)\right)=0
$$

In Ref. [6], in the infinite noncommutativity limit, bosonic D-branes were constructed explicitly only for those of even codimensions, viz. D23-, D21-, .. D1-branes but the rest, viz. D24-, D22-, .... D0-branes, were left out. In the commutative limit, all D p-branes are on equal footing, as they are constructed out of the tachyon field as localized energy lumps of both odd and even codimensions. It is highly unlikely that, for some mysterious reasons, the D-branes of odd codimensions are suppressed relative to those of even codimensions as noncommutativity is turned on. How then do the D-branes of odd codimensions arise in the infinite noncommutativity limit? In this Letter, we point out that, in both bosonic string and superstring theories, D-branes of odd codimensions can be constructed by applying a $\mathrm{U}(\infty)$ symmetry transformation. It has already been emphasized in [6] that the $U(\infty)$ symmetry arises as a gauge symmetry on the worldvolume of noncommutative D-branes. Moreover, the D-branes we will find are T-dual to the ones found in [6]. As such, our result may be interpreted as showing 3 that the T-duality, known to be a discrete (gauge) symmetry in compactified bosonic and Type II superstring theories, is actually a part of the $\mathrm{U}(\infty)$ gauge symmetry of the noncommutative D-branes.

We will begin with posing an issue concerning noncommutative $\mathrm{D} p$-branes in bosonic open

\footnotetext{
${ }^{2}$ Such solitons were constructed earlier in the context of $c=1$ matrix model and two-dimensional QCD [5].

${ }^{3}$ We are grateful to J.A. Harvey for emphasizing to us the importance of the interpretation and for further discussions.
} 
string theory. In doing so, we will bring up the puzzle concerning missing $\mathrm{D} p$-branes for $p=$ even more explicit. At level-zero truncation, the bosonic open string field theory is described by the action of the tachyon field $T$ :

$$
S_{0}=\frac{C}{G_{\text {st }}} \int d^{26} y \sqrt{-G}\left(\frac{1}{2} G^{\mu \nu} F(T) \partial_{\mu} T \partial_{\nu} T+\cdots-V(T)\right)_{\star},
$$

in which the tachyon potential $V(T)$ is taken of the form

$$
V(T)=-\frac{1}{2} \widetilde{T}^{2}+\frac{1}{3 T_{0}} \widetilde{T}^{3} \quad \text { where } \quad \widetilde{T}(x)=\exp \left[\ln \left(\frac{3 \sqrt{3}}{4}\right) \partial_{x}^{2}\right] T(x)
$$

with a maximum at $T_{0}$ and a minimum at $T=0$. The constant $C$ is related to the tension of the D25-brane $T_{25}$ and the closed string coupling parameter $g_{\mathrm{st}}$ as:

$$
C \frac{V\left(T_{0}\right)}{g_{\mathrm{st}}}=T_{25}
$$

and $G_{\mathrm{st}}, G_{\mu \nu}$ refers to the open string coupling and metric, respectively. The $\star$-subscript refers to the fact that field products are defined in terms of the noncommutative Moyal product:

$$
T(y) \star T(y)=\left.\exp \left(\frac{i}{2} \theta^{\mu \nu} \partial_{\mu}^{a} \partial_{\nu}^{b}\right) T\left(y_{1}\right) T\left(y_{2}\right)\right|_{y_{a}, y_{b} \rightarrow y} .
$$

Let us assume that noncommutativity is turned on along the $\mathbf{y}=\left(y^{1}, y^{2}\right)$ directions only and has the strength $\theta^{12}=\theta^{21} \equiv \theta$. We shall be mostly interested in the infinite noncommutativity limit: $\theta \rightarrow \infty$. Rescaling the $\mathbf{y}$-coordinates along the noncommutativity directions as $\mathbf{y}=\sqrt{\theta} \mathbf{x}$, both in the quadratic gradient term and the cubic tachyon interaction term Eq.(2), the action Eq.(11) can be recast as

$$
S_{0}=\frac{C}{G_{\mathrm{st}}} \theta \int d^{2} \mathbf{x} d^{24} y \sqrt{-G}\left(\frac{1}{2} G^{\mu \nu} F(T) \partial_{\mu} T \partial_{\nu} T-V(T)+\cdots\right)_{\star},
$$

where $\mu . \nu=0,3,4, \cdots, 25, V(T)$ refers to the tachyon potential in which $\widetilde{T}$ is simply set equal to $T$, and the ellipses denote subleading terms of order $\mathcal{O}(1 / \theta)$. The $\star$-subscript now refers to the rescaled Moyal product

$$
T(\mathbf{x}) \star T(\mathbf{x})=\left.\exp \left(\frac{i}{2}\left(\partial_{1}^{a} \partial_{2}^{b}-\partial_{1}^{b} \partial_{2}^{a}\right)\right) T\left(\mathbf{x}_{a}\right) T\left(\mathbf{x}_{b}\right)\right|_{\mathbf{x}_{a}, \mathbf{x}_{b} \rightarrow \mathbf{x}} .
$$

The equation of motion is then given by

$$
G^{\mu \nu} \partial_{\mu} \partial_{\nu} T-V_{\star}^{\prime}(T)=0
$$

which, in the static and translationally invariant case, reduces to

$$
V_{\star}^{\prime}(T)=-T+\frac{1}{T_{0}} T \star T=0 .
$$

\footnotetext{
${ }^{4}$ Later, we will readdress the precise nature of the $\theta \rightarrow \infty$ limit.
} 


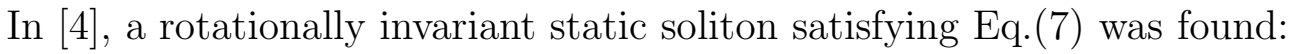

$$
T(\mathbf{x})=T_{0} \cdot 2 \exp \left(-\frac{r^{2}}{\theta}\right) \quad \text { where } \quad r^{2}=y_{1}^{2}+y_{2}^{2} .
$$

The tachyon field interpolates between $T=2 T_{0}$, twice of the value of the potential maximum, and $T=0$, the closed string vacuum. The solution thus describes an object of $(23+1)$ dimensional worldvolume, which can be interpreted [6] as a noncommutative version of the bosonic D23-brane.

As shown in [6], one can construct all other even codimensional D-branes by turning on $B$ fields along $\left(x^{1}, x^{2}\right),\left(x^{3}, x^{4}\right), \ldots,\left(x^{24-2 n}, x^{25-2 n}\right)$ subspaces. If the $B$-fields are all equal, then the resulting soliton may be identified with a noncommutative version of the bosonic D $2 n$-brane, for which the $\mathbf{r}^{2}$ is replaced by $\mathbf{r}^{2}=\left(x^{1}\right)^{2}+\cdots+\left(x^{25-2 n}\right)^{2}$. Apparently, the noncommutative D-branes constructed this way are only those with even codimensions. Where are the missing bosonic D-branes of odd codimensions?

To answer the question posed, let us recapitulate the systematics of constructing noncommutative solitons, following closely the works of [4] and [6]. The systematics is based on the so-called Moyal-Weyl correspondence - the equivalence between the configuration space of fields on a noncommutative space whose algebra is defined in terms of Moyal-product and the Hilbert space of Weyl-ordered operators in one-particle quantum mechanics.

Consider a scalar field $T(\mathbf{x})$ defined on a noncommutative plane with the associated Moyalproduct Eq.(6). The Fourier-mode function $\widetilde{T(\mathbf{k})}$ is defined by the equation

$$
T(\mathbf{x})=\int \frac{d^{2} \mathbf{k}}{(2 \pi)^{2}} \widetilde{T(\mathbf{k})} e^{i \mathbf{k} \cdot \mathbf{x}}
$$

Consider now operators $\widehat{x^{1}}, \widehat{x^{2}}$ satisfying the Heisenberg algebra

$$
\left[\widehat{x^{1}}, \widehat{x^{2}}\right]=i
$$

Built out of the operator algebra is an auxiliary Hilbert space $\mathcal{H}_{\theta}$ of an auxiliary one-particle quantum mechanics. Given an operator $\widehat{T}(\widehat{\mathbf{x}})$ acting on $\mathcal{H}_{\theta}$, one can define its Fourier-mode function as:

$$
\widehat{T}(\widehat{\mathbf{x}})=\int \frac{d^{2} \mathbf{k}}{(2 \pi)^{2}} \widetilde{T}(\mathbf{k}) e^{i \mathbf{k} \cdot \widehat{\mathbf{x}}}
$$

The Moyal-Weyl correspondence then refers to the equivalence between the $\star$-product algebra of $T(\mathbf{x})$ and the operator algebra of $\widehat{T}(\widehat{\mathbf{x}})$ under the condition that the Fourier mode functions are set equal:

$$
\widetilde{T(\mathbf{k})}=\widetilde{T(\mathbf{k})}
$$

\footnotetext{
${ }^{5}$ Refs. 4, 11, 12 provide useful reviews of the formalism in the context of noncommutative field theories and gauge theories.
} 
It then follows straightforwardly that

$$
\begin{aligned}
\widehat{T}(\widehat{\mathbf{x}}) \widehat{U}(\widehat{\mathbf{x}}) & \longleftrightarrow \quad T(\mathbf{x}) \star U(\mathbf{x}) \\
\operatorname{Tr} \widehat{T}(\widehat{\mathbf{x}}) \widehat{U}(\widehat{\mathbf{x}}) \cdots & \longleftrightarrow \quad \int \frac{d^{2} \mathbf{x}}{(2 \pi \theta)} T(\mathbf{x}) \star U(\mathbf{x}) \star \cdots .
\end{aligned}
$$

In particular, the equivalence implies that static solitons are obtained in the operator formulation as distribution of critical points of the tachyon potential among the set of one-dimensional projection operators in $\mathcal{H}_{\theta}$ - an important observation made in Ref. [4] and used in the context of D-branes in [6], [7] and [8].

Stated as above, the Moyal-Weyl correspondence does not specify at all what basis one needs to choose for the auxiliary Hilbert space $\mathcal{H}_{\theta}$. In fact, no specific Hamiltonian of the analog one-particle quantum mechanics is specified either. It is clear, however, that the choice of the basis should reflect additional information on the phase space labelled by $\mathbf{x}$ such as symmetries, presence/absence of boundaries, etc. As such, in what follows, we will consider two elementary choices of the basis - simple harmonic oscillator basis and plane-wave basis and propose to identify them with D-branes of codimension even and odd, respectively.

As the first choice, let us take the energy eigenbasis $\{|n\rangle, n=0,1, \cdots\}$ of a simple harmonic oscillator as the 'auxiliary' one-particle quantum mechanics. The basis is built out of creation and annihilation operators:

$$
a=\frac{1}{\sqrt{2}}\left(\lambda \widehat{x^{1}}+i \frac{1}{\lambda} \widehat{x^{2}}\right) \quad a^{\dagger}=\frac{1}{\sqrt{2}}\left(\lambda \widehat{x^{1}}-i \frac{1}{\lambda} \widehat{x^{2}}\right)
$$

where $\lambda$ is a free parameter. They are defined by the following action on the Hilbert space $\mathcal{H}_{\theta}$ :

$$
a|n\rangle=\sqrt{n}|n+1\rangle \quad a^{\dagger}|n\rangle=\sqrt{n+1}|n+1\rangle
$$

so that

$$
|n\rangle=\frac{a^{\dagger n}}{\sqrt{n !}}|0\rangle
$$

and

$$
|m\rangle\langle n|=: \frac{a^{\dagger m}}{\sqrt{m !}} e^{-a^{\dagger} a} \frac{a^{n}}{\sqrt{n !}}:
$$

The most general projection operator in the subspace spanned by the first $N$ eigenstates is given by

$$
\widehat{\mathbf{P}}=\sum_{m, n=0}^{N-1} C_{m n}|m\rangle\langle n| \quad \text { with } \quad \sum_{n} C_{m n} C_{n q}=C_{n q} \quad \text { and } \quad \sum_{m} C_{m m}=1 .
$$

\footnotetext{
${ }^{6}$ In the case of Eq.(2), the critical points consist of $\left\{0, T_{0}\right\}$.
} 
The conditions on $C_{m n}$ 's are imposed to ensure the projection operator properties: $\widehat{\mathbf{P}} \cdot \widehat{\mathbf{P}}=\widehat{\mathbf{P}}$ and $\operatorname{Tr} \widehat{\mathbf{P}}=1$. As a simple choice with 'zero' angular momentum (that is, a diagonal projection operator), let us take

$$
\widehat{\mathbf{P}}_{n}=|n\rangle\langle n| \quad(n=0,1,2, \cdots) .
$$

From Eq.(99), one easily finds the corresponding Fourier mode-function $\widetilde{\widehat{P}_{n}}(\mathbf{k})$ as:

$$
\widetilde{P_{n}(\mathbf{k})}=e^{-k^{2} / 4} L_{n}\left(k^{2} / 2\right), \quad \quad k^{2}=\left(k_{1}^{2}+k_{2}^{2}\right),
$$

where $L_{n}$ denotes the $n$-th order Laguerre polynomial. Using (10) and inserting the Fouriermode function into Eq.(8), one obtains the Weyl-Moyal (inverse) map $\widetilde{\mathbf{P}}_{n}(\mathbf{x})$ of the projection operator:

$$
\mathbf{P}_{n}(\mathbf{x})=2(-1)^{n} e^{-\tilde{r}^{2}} L_{n}\left(2 \tilde{r}^{2}\right) \quad \text { where } \quad \tilde{r}^{2}=\left(\lambda^{2} x_{1}^{2}+\frac{1}{\lambda^{2}} x_{2}^{2}\right) .
$$

From this the static noncommutative soliton of codimension-two can be constructed as $T(\mathbf{x})=$ $T_{0} \mathbf{P}_{n}(\mathbf{x})$. One can check straightforwardly that $\mathbf{P}_{n}(\mathbf{x})$ continues to satisfy the properties of the projection operator: $\mathbf{P}_{n}(\mathbf{x}) \star \mathbf{P}_{n}(\mathbf{x})=\mathbf{P}_{n}(\mathbf{x})$ and $\int d^{2} \mathbf{x} /(2 \pi \theta) \mathbf{P}_{n}(\mathbf{x})=1$. The solution Eq.(7) corresponds to (a) the choice of $n=0$ among all possible codimension-two solitons in Eq.(12) and (b) the rotationally symmetric choice of the $\lambda$-parameter, $\lambda=1$.

As the second choice, we will take projection operators related to the 'plane-wave' basis $\{|p\rangle\}$ of the Hilbert space $\mathcal{H}_{\theta}$. In $\mathcal{H}_{\theta}$, the 'plane-wave' basis is defined by

$$
\widehat{x^{2}}|p\rangle=p|p\rangle \quad\langle q \mid p\rangle=\frac{1}{\sqrt{2 \pi}} e^{i p q}
$$

and

$$
\left\langle p \mid p^{\prime}\right\rangle=\delta\left(p, p^{\prime}\right) \quad \int \frac{d p}{2 \pi}|p\rangle\langle p|=\mathbf{I} .
$$

The most general projection operator is given by

$$
\widehat{\mathbf{P}}=\int \frac{d p d p^{\prime}}{(2 \pi)^{2}} C\left(p, p^{\prime}\right)|p\rangle\left\langle p^{\prime}\right| \quad \text { with } \quad \int \frac{d q}{2 \pi} C(p, q) C\left(q, p^{\prime}\right)=C\left(p, p^{\prime}\right) \quad \text { and } \quad \int \frac{d p}{2 \pi} C(p, p)=1 .
$$

A simple choice for a 'monochromatic' projection operator would be

$$
\widehat{\mathbf{P}}_{\widetilde{p_{0}}}=\left|\widetilde{p_{0}}\right\rangle\left\langle\widetilde{p_{0}}\right| \text {. }
$$

To be precise, $\left|\widetilde{p_{0}}\right\rangle$ is to be taken not as a strict monochromatic wave of momentum $\widetilde{p_{0}}$ but as a wave-packet built around mean-value of the momentum $\widetilde{p_{0}}$ and nonzero, finite variance. These conditions are necessary in order to ensure $\left\langle\widetilde{p_{0}} \mid \widetilde{p_{0}}\right\rangle=1$ and to avoid complications associated with delta-function (instead of square-integrable) normalizability as well as subtle 
issues concerning infinite noncommutativity and large deformation, which will be elaborated later. For definiteness, we may define $\left|\widetilde{p_{0}}\right\rangle$ operationally in terms of the following wave-packet:

$$
\left|\widetilde{p_{0}}\right\rangle=\int \frac{d p}{\sigma \sqrt{\pi}} \exp \left(-\left(p-\widetilde{p_{0}}\right)^{2} / \sigma^{2}\right)|p\rangle .
$$

To obtain the monochromatic plane wave state, we let $\sigma \rightarrow 0$ in the end with a suitably defined limit procedure.

It is straightforward to compute, as before, the Weyl-Moyal (inverse) map $\mathbf{P}_{p_{0}}(\mathbf{x})$ of the projection operator Eq.(13). The result is

$$
\mathbf{P}_{\widetilde{p_{0}}}(\mathbf{x})=2 \exp \left(-\frac{\left(x_{2}-\widetilde{p_{0}}\right)^{2}}{\sigma^{2}}-\sigma^{2} x_{1}^{2}\right) .
$$

One easy finds that, as $\sigma \rightarrow 0$, the above function approaches

$$
\mathbf{P}_{\widetilde{p_{0}}}(\mathbf{x})=\frac{1}{2 \pi R_{1}} \delta\left(x_{2}-\widetilde{p_{0}}\right)
$$

consisting of a delta-function in $x^{2}$-direction while all dependence on $x^{1}$-direction disappears. Here, $R_{1}$ is a suitably large radius of the $x^{1}$-direction and the factor $1 /\left(2 \pi R_{1}\right)$ is to take care of the requisite normalization condition, where the 'box cut-off' $R_{1}$ is related to the 'Gaussian cut-off' $\sigma$ as $R_{1} \sim 1 / \sqrt{\sigma}$.

From Eq. (15), one obtains a soliton of codimension-one as $T(\mathbf{x})=T_{0} \mathbf{P}_{\widetilde{p_{0}}}(\mathbf{x})$. The profile of this soliton is very different from that of the circularly symmetric soliton discussed previously. Eq.(15) represents, on the noncommutative plane, a kink rather than a vortex. We will shortly identify it as a D24-brane formed out of tachyon condensation on the D25-brane worldvolume.

Before doing so, we should point out the underlying philosophy of our construction. As mentioned before, various choices of orthonormal bases of $\mathcal{H}_{\theta}$ are related to one another by $\mathrm{U}(\infty)$ transformations. As shown in [6], the $\mathrm{U}(\infty)$ transformations are in fact gauge transformations on the D-brane worldvolume. A one-dimensional projection operator is given by $|\psi\rangle\langle\psi|$ for any arbitrary state $|\psi\rangle$ in $\mathcal{H}_{\theta}$. What we found in the above construction is that the Weyl-Moyal map corresponding to different choices of $|\psi\rangle$ takes generically very different shapes on the noncommutative plane, and are related to each other by gauge transformations.

In fact, different choices of the basis $|\psi\rangle$ are characterized by the Grassmannian associated with a single soliton:

$$
\mathcal{M}_{1}=\frac{\mathrm{U}(\infty)}{\mathrm{U}(\infty-1) \times \mathrm{U}(1)} .
$$

For example, the various values of $\lambda$ in Eq.(11) correspond to a one-parameter 'squeezed state' subspace in the above manifold. The corresponding tachyon solutions are related to one another by $\mathrm{U}(\infty)$ gauge transformations

$$
T(\mathbf{x}) \longrightarrow U(\mathbf{x}) \star T(\mathbf{x}) \star U^{\dagger}(\mathbf{x})
$$


The $\mathrm{U}(\infty)$ gauge transformations clearly respect the projection operator properties: $(\mathbf{P} \star$ $\mathbf{P})(\mathbf{x})=\mathbf{P}(\mathbf{x})$ and $\int d \mathbf{x} /(2 \pi \theta) \mathbf{P}(\mathbf{x})=1$; they also preserve rank of the projection operator so that all points of a gauge orbit correspond to the same rank. Remarks similar to these and the possibility of squeezing deformation have been made in [4, 6]; we have found here an explicit application of these ideas to construct bosonic D24 branes.

As a concrete illustration of the $\mathrm{U}(\infty)$ gauge transformation, we now map the codimensiontwo profile Eq.(12) to the codimension-one profile Eq.(15). This is achieved by recalling that the parameter $\lambda$ is associated with the squeezing deformation, an aspect discussed already in 清. One finds that the $\lambda \rightarrow 0$ limit of Eq.(12) reduces exactly to Eq.(15) for the choice $\widetilde{p_{0}}=0$. In fact, in this limit, the squeezing deformation parameter $\lambda$ plays precisely the same role as the regularization parameter $\sigma$ in Eq.(14). The opposite squeezing limit $\lambda \rightarrow \infty$ yields again the same state as Eq.(15) but with $x^{1} \rightarrow x^{2}, x^{2} \rightarrow-x^{1}$ which amounts to a rotation in the noncommutative plane. The moduli space of the squeezing deformation is parametrized by $\lambda \in[0,1]$. We have thus shown that the parameter $\lambda$ spans a one-parameter trajectory in $\mathcal{M}_{1}$ associated with the squeezed state and the two extreme endpoint configurations represent geometrically codimension-one as $\lambda \rightarrow 0$ and codimension-two as $\lambda \rightarrow 1$, respectively.

At this point, we would like to discuss an important subtlety $\square$ in applying the $\mathrm{U}(\infty)$ gauge transformation and obtaining the codimension-one, kink configuration. Recall that the action Eq.(5) was obtained by dropping terms involving derivatives along the noncommutative directions - both in the quadratic gradient term and in the cubic interaction term — by taking $\theta \rightarrow \infty$ limit. On the other hand, in applying the squeezing deformation over the Grassmannian $\mathcal{M}_{1}$ and letting $\lambda \rightarrow 0$, as described above, each derivative along the noncommutative directions is amplified by a factor $1 / \lambda$. Certainly, to retain the utility of the infinite noncommutativity and construction of the noncommutative D-branes via the Moyal-Weyl correspondence, one ought to take a controlled limit keeping $\theta_{\text {eff }} \equiv \lambda^{2} \theta$ fixed, while letting $\theta \rightarrow \infty$ and $\lambda \rightarrow 0$. In order to suppress all the terms involving derivatives along the noncommutative directions, it is necessary to take $\theta_{\text {eff }} \gg 1$. In fact, one easily finds that, once the squeezing deformation is

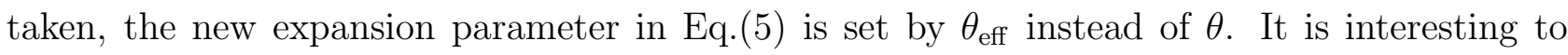
consider whether such subtleties involving large spatial derivatives arise in some other parts o f the Grassmannian $\mathcal{M}_{1}$ as well. We expect that the standard large- $\theta$ limit can be taken only in the interior of the Grassmannian $\mathcal{M}_{1}$, but not along the boundary. As the boundary is approached, the large- $\theta$ constructions would in general require a suitably controlled scaling such as the one we have defined above.

Having obtained a codimension-one profile, we are naturally led to identify the soliton Eq.(15) with a noncommutative version of the bosonic D24-brane. To ascertain this identifica-

\footnotetext{
${ }^{7}$ We would like to thank E. Witten for pointing out to us the importance of this issue, which has already been discussed in [8].
} 
tion, we will now calculate the tension of the codimension-one soliton and compare it with the known string theory result. For a static solution, the action is simply the time interval times the mass, so the soliton mass can be equivalently calculated out of the action. We will calculate the action Eq.(5) for the tachyon field $T_{\text {sol }}(\mathbf{x}, y)=T_{0} \mathbf{P}_{\widetilde{p_{0}}}(\mathbf{x})$ where $\mathbf{P}_{p_{0}}(\mathbf{x})$ is given by Eq.(15). To be specific, we will take the closed string background as

$$
\begin{aligned}
d s^{2} & =\theta\left(R_{1}^{2} d x_{1}^{2}+d x_{2}^{2}\right)+d y_{\mu} d y^{\mu} \\
e^{\phi} & =g_{\mathrm{st}} \\
B_{2} & =B d x^{1} \wedge d x^{2}
\end{aligned}
$$

From the definition of the open string coupling $G_{\text {st }}$ and the open string metric $G_{\mu \nu}$ [3], one finds (cf. [6]) in the large $\theta$ limit

$$
G_{\mathrm{st}}=g_{\mathrm{st}} \frac{\sqrt{G}}{2 \pi \ell_{\mathrm{st}}^{2} B} .
$$

Using the results that

$$
\begin{aligned}
V\left(T_{\text {sol }}(\mathbf{x}, y)\right) & =V\left(T_{0}\right) \mathbf{P}_{\widetilde{p_{0}}}(\mathbf{x}) \\
\frac{C}{g_{\text {st }}} V\left(T_{0}\right) & =T_{25} \\
\int \frac{d^{2} \mathbf{x}}{2 \pi \theta} \mathbf{P}_{\widetilde{p_{0}}}(\mathbf{x}) & =1 \\
1 / B & =\theta,
\end{aligned}
$$

we find that the action Eq.(5) is evaluated to

$$
S=T_{25}\left(2 \pi \ell_{\mathrm{st}}\right)^{2} \int d t d y^{3} \ldots d y^{25} .
$$

This agrees with the action of the codimension-two soliton evaluated in [6], reflecting the $\mathrm{U}(\infty)$ gauge invariance mentioned above, $\int d \mathbf{x} /(2 \pi \theta) U(\mathbf{x}) \star \mathbf{P}_{\widetilde{p_{0}}}(\mathbf{x}) \star U^{\dagger}(\mathbf{x})=\int d \mathbf{x} /(2 \pi \theta) \mathbf{P}_{\widetilde{p_{0}}}(\mathbf{x})=1$.

In [6], the action Eq.(18) and the corresponding tension were interpreted as those of a D23brane extending along $y^{3}, \ldots, y^{25}$. The symmetries of our codimension-one soliton are those of a 24-brane, extending along $x^{1}, y^{3}, \ldots, y^{25}$ directions. We will now show that, it is actually a D24-brane living in the T-dual geometry where T-duality is performed along the $x^{1}$ direction. The closed string geometry T-dual to Eq.(16) is given by

$$
\begin{aligned}
\widetilde{d s}^{2} & =\frac{1}{R_{1}^{2}} d x_{1}^{2}-\frac{2 B}{R_{1}^{2}} d x_{1} d x_{2}+\left(1+\frac{B^{2}}{R_{1}^{2}}\right) d x_{2}^{2}+d y_{\mu} d y^{\mu} \\
e^{\widetilde{\phi}} & =\widetilde{g_{\mathrm{st}}}=g_{\mathrm{st}} \ell_{\mathrm{st}} / R_{1} \\
\widetilde{B} & =B .
\end{aligned}
$$

Our assertion would follow if the worldvolume action of a D24-brane extended along $x^{1}, y^{3}, \cdots, y^{25}$ in the T-dual background Eq.(19) is exactly the same as the action obtained from the tachyon 
soliton of codimension-one, Eq.(18). Begin with noting that the pull-back of the T-dual geometry on the D24-brane worldvolume is given by

$$
\widehat{d s}^{2}=\frac{1}{R_{1}^{2}} d x_{1}^{2}+d y_{\mu} d y^{\mu},
$$

while pull-back of the $B$-field on the worldvolume vanishes. The worldvolume action of a D24-brane is therefore reduced to

$$
S_{\mathrm{DBI}}=\widetilde{T_{24}} \int d t d x^{1} d x^{3} \ldots d x^{25} \sqrt{\widehat{g}}
$$

where $\widetilde{T_{24}}$ is the tension of a T-dualized D24-brane, given by

$$
\widetilde{T_{24}}=T_{24} g_{\mathrm{st}} / \widetilde{g_{\mathrm{st}}}=T_{24} R_{1} / \ell_{\mathrm{st}}
$$

Using the fact that $\int d x^{1} \sqrt{\widehat{g}_{11}}=2 \pi / R_{1}$, we get

$$
S_{\mathrm{DBI}}=T_{24}\left(2 \pi \ell_{\mathrm{st}}\right) \int d t d x^{3} \ldots d x^{25}
$$

This is exactly the same as Eq.(18), as

$$
T_{24}=2 \pi \ell_{\mathrm{st}} T_{25}
$$

Hence, we have demonstrated that the codimension-one soliton Eq.(15) is indeed the noncommutative version of D24-brane. Moreover, as in the D23-brane case [6], we have also found that the exact value of the D24-brane tension (T-dualized) has come out of level-zero truncation.

Putting all the above together, we interpret the result as follows. The noncommutative D24-brane on a circle we have constructed exhibits precisely the same properties as a noncommutative D23-brane distributed on a T-dual circle. As both D-branes have the same rank and are unitarily related to each other by (a subset of) $\mathrm{U}(\infty)$ gauge transformation on $\mathcal{M}_{1}$, we have essentially shown that the T-duality, which is known to be a gauge symmetry in bosonic string theory, is in fact a part of $\mathrm{U}(\infty)$ gauge symmetry, at least in the large noncommutativity limit. While we have presented the noncommutative codimension-one solitons for bosonic open string theory, the same analysis goes through for noncommutative version of the non-BPS D-branes in Type II string theories. It, however, entails an interesting variation of the theme. The fact that bosonic $\mathrm{D} p$-branes of both even and odd $p$ constructed as above are related to each other by $\mathrm{U}(\infty)$ transformations does not contradict any known symmetry principle. However, in superstring theory, for example Type IIA, non-BPS $p$ branes arise only for odd $p$ (similarly for Type IIB only even $p$ ). So, there clearly cannot exist a $\mathrm{U}(\infty)$ symmetry within either IIA or IIB superstring theory, which relates non-BPS D- $p$ branes of different worldvolume dimensions. What, then, is the interpretation of the $\mathrm{U}(\infty)$ gauge transformation? We will now show that 
the transformation relates non-BPS noncommutative D-branes in Type IIA/IIB to those in Type IIB/IIA via the T-duality and hence offers a new kind of D-brane descent relations.

Recall that in the commutative limit, Sen has given descent relations among D-branes via worldvolume kinks or vortices formed out of tachyon condensation. The noncommutative Dbrane descent relations we will present are different from those of Sen's. Moreover, we will find that the new series of descent relations provides a firmer support to our interpretation regarding the $\mathrm{U}(\infty)$ gauge transformation as a T-duality between Type IIA and IIB strings through an analysis of the Chern-Simons coupling and Ramond-Ramond charges, an aspect which was not available for bosonic D-brane setup.

Let us first recollect Sen's descent relations in the commutative limit. Begin with nonBPS D2p-branes in Type IIA string or $\mathrm{D}(2 p-1)$-branes in Type IIB string. These non-BPS branes are unstable, as represented by a tachyon field on the worldvolume. The tachyon is a real-valued field and it can condense in the form of a topological kink on the worldvolume of the non-BPS D-brane. As proven by Sen, the kink turns out precisely the same as BPS Dbrane. Thus, in Sen's descent relation, in Type IIA string, BPS D $(2 p-1)$-brane can be formed out of kink formation on the worldvolume of non-BPS $\mathrm{D}(2 p)$-brane and, in Type IIB string, BPS D $(2 p-2)$-brane can be formed out of kink formation on the worldvolume of non-BPS $\mathrm{D}(2 p-1)$-brane.

In infinitely strong noncommutative limit, solitons formed out of tachyon condensation can come with two varieties: (1) codimension-two solitons studied in [6, 7] or (2) codimension-one solitons proposed in the present paper.

For the case (1), even though the solitons are objects of codimension-two, they ought to be identified with circular ring of codimension-one, BPS object. This is because, as shown in [7], the Chern-Simons term on non-BPS D $p$-brane involves a coupling

$$
\frac{1}{2 T_{0}} \int_{M_{p+1}} d T \wedge C_{p}^{\mathrm{RR}}
$$

where $C_{p}^{\mathrm{RR}}$ is the Ramond-Ramond $p$-form potential. Inserting the noncommutative tachyon profile $T_{\text {sol }}(\mathbf{r})=T_{0} \mathbf{P}_{0}(r)$, one finds that the above coupling yields

$$
\frac{1}{2 T_{0}} \int_{M_{p+1}} T_{0} d \mathbf{P}_{0}(r) \wedge C_{p}^{\mathrm{RR}}=\frac{1}{2} \int_{M_{p+1}} \mathbf{P}_{0}^{\prime}(r) d r \wedge C_{p}^{\mathrm{RR}}
$$

Using the fact that $\int d r \mathbf{P}_{0}^{\prime}(r)=-\mathbf{P}_{0}(0)=-2$, one gets precisely $-\int_{M_{p}} C_{p}^{\mathrm{RR}}$, implying that the soliton carries (minus) one unit of the Ramond-Ramond charge, viz. BPS $\mathrm{D}(p-1)$-brane. As the object is circularly symmetric, a natural interpretation of the codimension-two soliton would be that it is a ring of the $\operatorname{BPS} \mathrm{D}(p-1)$-brane on the noncommutative plane. Thus, one can say that the codimension-two soliton formation is nothing but noncommutative counterpart of the Sen's kink formation. 
For the case (2), we will now argue that the soliton ought to be interpreted as the non-BPS $\mathrm{D}(p-1)$-brane of the T-dual, Type IIA/IIB string. To see this, let us evaluate the Chern-Simons coupling (21) for the codimension-one soliton profile $T_{\text {sol }}(\mathbf{r})=T_{0} \mathbf{P}_{\widetilde{p}_{0}}(\mathbf{x})$. We get

$$
\frac{1}{2 T_{0}} \int_{M_{p+1}} \frac{T_{0}}{R_{1}} d \delta\left(x^{2}\right) \wedge C_{p}^{\mathrm{RR}}=\frac{1}{2 R_{1}} \int_{M_{p+1}} \delta^{\prime}\left(x^{2}\right) \wedge C_{p}^{\mathrm{RR}} .
$$

In the present case, unlike the codimension-two solitons, $\int d x^{2} \delta^{\prime}\left(x^{2}\right)=0$ and hence the above Chern-Simons coupling vanishes. This shows that the codimension-one soliton carries no $\operatorname{BPS}(p-1)$-brane charge at all. Clearly, this should be considered as a non-BPS $(p-1)$-brane which does not carry a RR charge. Furthermore such a non-BPS brane cannot be in the original IIA/B theory since that contains non-BPS $p$-branes. This implies that one must consider this non-BPS $(p-1)$ as living in the T-dual geometry (T-dualized along $x^{1}$ ), a fact that we found in the bosonic context by an explicit computation of the tension. Furthermore, repeating the earlier analysis in the bosonic string context [6], one discovers that tachyon fluctuation $\delta T\left(x^{2}\right)$ induces a coupling of the form

$$
\frac{1}{2 T_{0}} \int_{M_{p}} d \delta T \wedge C_{p-1}^{\mathrm{RR}}
$$

This is indeed the correct form of the Chern-Simons coupling for a non-BPS D $(p-1)$-brane localized at a point along the T-dualized $\widetilde{x^{1}}$-direction. Again, we have shown that the T-duality between Type IIA and IIB superstring theories, which is known as a discrete symmetry, is part of the $\mathrm{U}(\infty)$ gauge symmetry arising in the large noncommutativity limit.

We are grateful to M.R. Douglas and S.R. Wadia for useful discussions, and J.A. Harvey and E. Witten for important suggestions. We acknowledge warm hospitality of Theory group at CERN during this work.

\section{References}

[1] A. Connes, M.R. Douglas and A. Schwartz, J. High-Energy Phys. 9802 (1998) 003, hep-th/9711162.

[2] M.R. Douglas and C.M. Hull, J. High-Energy Phys. 9802 (1998) 008, hep-th/9711165.

[3] N. Seiberg and E. Witten, J. High-Energy Phys. 9909 (1999) 032, hep-th/9908142.

[4] R. Gopakumar, S. Minwalla and A. Strominger, J. High-Energy Phys. 0005 (2000) 020, hep-th/0003160.

[5] A. Dhar, G. Mandal and S. R. Wadia, Mod. Phys. Lett. A7 3129 (1992), hep-th/9207011;

A. Dhar, G. Mandal and S. R. Wadia, Phys. Lett. B329 15 (1994), hep-th/9403050. 
[6] J.A. Harvey, P. Kraus, F. Larsen and E.J. Martinec, J. High-Energy Phys. 0007 (2000) 042, hep-th/0005031.

[7] K. Dasgupta, S. Mukhi and G. Rajesh, J. High-Energy Phys. 0006 (2000) 022, hep-th/0005006.

[8] E. Witten, "Noncommutative Tachyons and String Field Theory", hep-th/0006071.

[9] A. Sen, J. High-Energy Phys. 9912 (1999) 027, hep-th/9911116.

[10] J.A. Harvey and P. Kraus, J. High-Energy Phys. 0004 (2000) 012, hep-th/0002117.

[11] D. J. Gross and N. Nekrasov, "Monopoles and Strings in Non-Commutative Gauge Theory", hep-th/0005204.

[12] L. Alvarez-Gaume and S. R. Wadia, "Gauge Theory on a Quantum Phase Space", hep-th/0006219. 